\title{
Activation of cannabinoid system in anterior cingulate cortex and orbitofrontal cortex modulates cost-benefit decision making
}

\author{
Abbas Khani • Mojtaba Kermani • Soghra Hesam • \\ Abbas Haghparast • Enrike G. Argandoña • \\ Gregor Rainer
}

\begin{abstract}
Despite the evidence for altered decision making in cannabis abusers, the role of the cannabinoid system in decision-making circuits has not been studied. Here, we examined the effects of cannabinoid modulation during costbenefit decision making in the anterior cingulate cortex (ACC) and orbitofrontal cortex (OFC), key brain areas involved in decision making. We trained different groups of rats in a delay-based and an effort-based form of cost-benefit T-maze decision-making task. During test days, the rats received local injections of either vehicle or ACEA, a cannabinoid type-1 receptor (CB1R) agonist in the ACC or OFC. We measured spontaneous locomotor activity following the same treatments and characterized CB1Rs localization on different neuronal populations within these regions using immunohistochemistry. We showed that CB1R activation in the ACC impaired
\end{abstract}

A. Khani - G. Rainer

Visual Cognition Laboratory, Department of Medicine, University of Fribourg, Chemin du Musee 5, CH 1700 Fribourg, Switzerland

\footnotetext{
A. Khani $\cdot$ G. Rainer $(\bowtie)$

Center for Cognition, University of Fribourg, Chemin du Musee 5, CH 1700 Fribourg, Switzerland

e-mail: gregor.rainer@unifr.ch
}

M. Kermani $\cdot$ S. Hesam · A. Haghparast

Neuroscience Research Center, Shahid Beheshti University of Medical Sciences, Tehran, Iran

E. G. Argandoña

Department of Medicine, University of Fribourg, Fribourg, Switzerland

\section{E. G. Argandoña}

Laboratory of Experimental Neuroscience (LaNCE), University of the Basque Country (UPV/EHU), Leioa, Spain

\section{Kermani}

Visual \& Cognitive Neuroscience Laboratory, Dept of Optometry \& Vision Science, University of Melbourne, Parkville, Australia decision making such that rats were less willing to invest physical effort to gain high reward. Similarly, CB1R activation in the OFC induced impulsive pattern of choice such that rats preferred small immediate rewards to large delayed rewards. Control tasks ensured that the effects were specific for differential cost-benefit tasks. Furthermore, we characterized widespread colocalizations of CB1Rs on GABAergic axonal ends but few colocalizations on glutamatergic, dopaminergic, and serotonergic neuronal ends. These results provide first direct evidence that the cannabinoid system plays a critical role in regulating cost-benefit decision making in the ACC and OFC and implicate cannabinoid modulation of synaptic ends of predominantly interneurons and to a lesser degree other neuronal populations in these two frontal regions.

Keywords Anterior cingulate cortex · Cannabinoid . Decision making · Orbitofrontal cortex · T-maze

\section{Introduction}

Cannabinoids are the main active ingredients of marijuana, an illicit drug in many countries that is widely used as a recreational substance due to its psychoactive effects. There is considerable evidence implicating the use of various cannabis derivatives in altered cognition and decision making, including risk taking behavior, irrational (or excessive) gambling, as well as impulsive and apathetic patterns of choice. This evidence comes mainly from human studies (Dougherty et al. 2013; Grant et al. 2012; Hermann et al. 2009; Lane et al. 2005; Moreno et al. 2012; Solowij et al. 2012; Whitlow et al. 2004) and related work in animals (Boomhower et al. 2013; Egerton et al. 2006; Egerton et al. 2005; Mishima et al. 2002) that largely concurs with the results of the human studies. The role of cannabinoid signaling in decision-making pathways and the involvement of cannabinoid receptors in frontal cortical 
circuits of decision making have not received much attention to date.

Endocannabinoids have emerged as fundamental modulators of synaptic function regulating a wide range of brain functions including feeding behavior (Engeli 2012), memory (Hampson et al. 2011; Jacob et al. 2012), stress responses (Ganon-Elazar and Akirav 2009), motor control (Dubreucq et al. 2013), and pain processing (Lee et al. 2013; Martin et al. 1999). Cannabinoids predominantly exert their modulatory effects in a retrograde manner by binding to cannabinoid receptors on presynaptic terminals (Castillo et al. 2012; Kano et al. 2009). Cannabinoid type1 receptors (CB1Rs) are highly abundant in many brain regions including frontal areas and are thought to mediate most of the brain cannabinoid system functions (Egertova and Elphick 2000; Kano et al. 2009; Katona and Freund 2008; Tsou et al. 1998). CB1Rs are densely distributed in cerebral cortex with the highest abundance in the cingulate cortex and association cortical regions of the frontal lobe (Eggan and Lewis 2007; Mailleux and Vanderhaeghen 1992; Svizenska et al. 2008). For example, Eggan and Lewis demonstrated a higher density of CB1Rs in the cingulate cortex and associative prefrontal regions compared to primary sensory and motor cortices (2007). The anterior cingulate cortex (ACC) and orbitofrontal cortex (OFC), two key brain regions with high prevalence of CB1Rs (Eggan and Lewis 2007; Filbey et al. 2010), play a cardinal role in information processing during decision-making tasks (Bechara et al. 2000; Kennerley et al. 2011). It has been shown that these two brain regions have partially dissociable roles in several dimensions of decision making (Khani 2014; Lee et al. 2007; Rushworth et al. 2007) which include the evaluation of the expected benefits as well as costs associated with each potential choice of action during a decision. In decision-making paradigms, costs typically take the form of delay costs, corresponding to a time delay, during which the subject has to wait for an expected benefit, or effort costs, where subject must expend physical effort to gain access to an expected benefit. A recent lesion study has shown that, indeed, the ACC and OFC are involved in the processing of distinct decision costs during decision making (Rudebeck et al. 2006). While ACC-lesioned rats exhibited similar decisions as control animals for delay costs, they exhibited pronounced deficits during decisions involving effort costs. The converse was true in the OFC: OFC-lesioned rats were highly impaired on decisions involving delay costs, but not different from control animals in decisions based on the effort costs. A human neuroimaging study showed a similar double dissociation between OFC and ACC in terms of processing of delay and effort decision costs (Prevost et al. 2010).
The role of different neurotransmitters/modulators in modulating neural circuits of decision making in the frontal lobe remains poorly understood. We aimed here to explore the modulatory effects mediated by cannabinoids on effortbased and delay-based decision making in the ACC and OFC. To address this, we used microinjections of cannabinoid agents into the ACC and OFC during both versions of the cost-benefit decision task. This allowed us to investigate whether the double dissociation between the ACC and OFC in the processing of distinct decision costs holds true following cannabinoid system activation in these same brain structures. It is noteworthy that cannabinoid-induced disturbances in the frontal cortex functions have been described for both acute as well as chronic cannabinoid administration. The aim of the current study is to shed light on the acute modulatory role of the cannabinoid system in these areas during decision making and long-term changes resulting from chronic cannabinoid consumption may rely on different mechanisms that need to be studied separately. In addition to the behavioral experiments, we characterized the neuronal populations involved using double-immunohistochemistry of the CB1R and selected neuronal markers. We also performed control experiments to demonstrate that these impairments are indeed costbenefit decision-making impairments and are not the result of memory or locomotor confounds.

\section{Materials and methods}

\section{Subjects}

A total of 54 male Wistar rats (Pasteur Institute, Iran or Janvier, Europe) were used as subjects. The rats were 8 weeks old at their arrival to the animal facility. Rats were housed in groups of three per cage under standard conditions in a temperature-controlled room and maintained on a standard 12-h light/dark cycle (lights on at 0700 hours). Water was available ad libitum. The animals were handled on a daily basis and food was adjusted for initial body weights of about $90 \%$ of the free feeding weight during the beginning of the behavioral experiment (270-300 g) and after this a controlled weight gain of about 6-12 g per week. All animals were naïve to the current tests and had no prior experience in any behavioral experiment. Main behavioral experiments, histological analyses and sample preparations for microscopy, and confocal microscopy were carried out at Shahid Beheshti Medical University, University of Fribourg, and University of the Basque Country, respectively. All experiments were executed in compliance with applicable Swiss regulations and the European Community Council Directive (2010/63/EU), or in accordance with the Guide for the Care and Use of Laboratory Animals (National Institutes of Health Publication No. 80-23, revised 1996) in Iran. 
Apparatus

T-mazes were constructed with parameters adopted from the study by Denk et al. (2005). The wooden mazes had three arms each $60 \mathrm{~cm}$ long, $10 \mathrm{~cm}$ wide, and $40 \mathrm{~cm}$ high. The floor and lower parts of the inner walls of the maze were painted with a transparent oil-based color to maintain a homogenous appearance of the maze. Metal food wells, $3 \mathrm{~cm}$ in diameter, were placed at the end of the goal arms $2 \mathrm{~cm}$ above the maze floor. For experiment 1, three-dimensional triangular barriers made of mesh wire with different heights $(5-30 \mathrm{~cm})$ were used in the midpoint of the high-reward goal arm to introduce different levels of physical effort cost in different stages of training. For experiment 2, four retractable doors were built in the goal arms of the maze. One door was placed just before the food well at each arm, $5 \mathrm{~cm}$ from the end of the arm and the other after the entrance into each arm, $12.5 \mathrm{~cm}$ from the entrance point (Fig. 1). These doors were used to delay the access of the animals to rewards in delay-based decisionmaking task. Furthermore, there were grooves at the beginning of the entrance to each goal arm in both mazes, where a barrier of $10 \mathrm{~cm}$ width and $40 \mathrm{~cm}$ height could be placed on certain trials to force the animal to go to one of the goal arms ("forced" trials). The maze was placed $1 \mathrm{~m}$ above the room floor and a video camera was mounted at the top to monitor animal behavior.

\section{Behavioral training}

Rats were trained to perform T-maze decision-making tasks with a differential cost (short vs. long waiting time or low vs. high effort) and reward (small vs. large reward amount) in the two arms of the maze. Figure $1 \mathrm{C}$ shows the sequence of training and experimental stages. With some slight differences, the procedure was adopted from the previous work (Denk et al. 2005; Rudebeck et al. 2006). Briefly, the rats were handled and weighed every day during the first week following their arrival. About 1 week after the beginning of food scheduling, the animals were habituated to the maze. For 3 days, the animals were allowed to explore the maze for

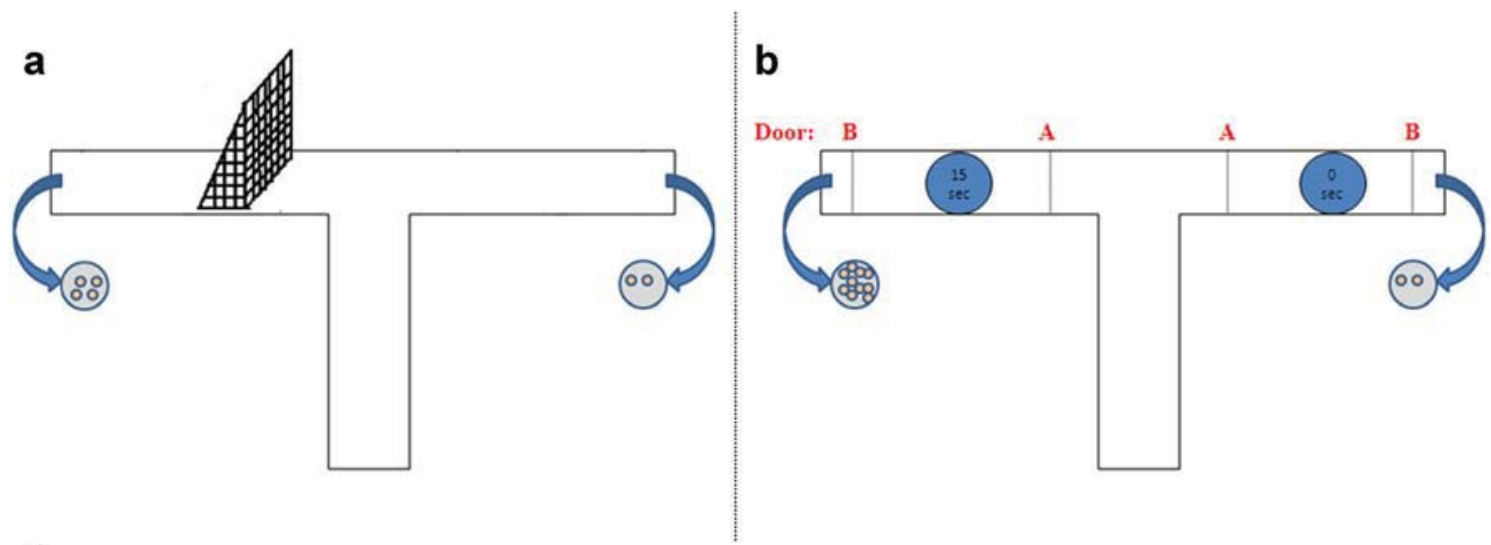

C

\begin{tabular}{|c|c|c|c|c|c|c|c|c|c|}
\hline \multirow[t]{2}{*}{$\begin{array}{l}\text { 1. Initial } \\
\text { Habituation } \\
\text { (14 days) }\end{array}$} & \multicolumn{2}{|c|}{$\begin{array}{l}\text { 2. Stages of } \\
\text { training with } \\
\text { increasing } \\
\text { cost } \\
\text { (25-35 days) }\end{array}$} & $\begin{array}{l}\text { 3. Surgery } \\
\text { and post- } \\
\text { operative } \\
\text { recovery } \\
(\sim 14 \text { days })\end{array}$ & \begin{tabular}{|c}
4. \\
Establishment \\
and \\
consolidation \\
of pre-surgery \\
performance \\
(12-15 days)
\end{tabular} & $\begin{array}{l}\text { 5. First } \\
\text { test day } \\
\text { with local } \\
\text { infustion } \\
\text { of either } \\
\text { CB1R } \\
\text { agonist or } \\
\text { vehicle }\end{array}$ & $\begin{array}{l}\text { 6. Free } \\
\text { behavior } \\
\text { (3 days) }\end{array}$ & \multicolumn{2}{|c|}{$\begin{array}{l}\text { 7. Second } \\
\text { test day with } \\
\text { counter- } \\
\text { balanced } \\
\text { infustion of } \\
\text { either vehicle } \\
\text { or CB1R } \\
\text { agonist }\end{array}$} & $\begin{array}{l}\text { 8. Free } \\
\text { behavior } \\
\text { ( } 3 \text { days) }\end{array}$ \\
\hline & $\begin{array}{l}\text { 9. Third } \\
\text { test day } \\
\text { infustion } \\
\text { of CB1R } \\
\text { antagonist }\end{array}$ & $\begin{array}{r}10 . \\
\text { fo } \\
\text { con } \\
\text { wit } \\
(10-\end{array}$ & $\begin{array}{l}\text { Training } \\
\text { or the } \\
\text { trol task } \\
\text { h equal } \\
\text { costs } \\
12 \text { days) }\end{array}$ & $\begin{array}{l}\text { 11. First test } \\
\text { day during } \\
\text { equal cost task } \\
\text { local infustion } \\
\text { of either } C B 1 R \\
\text { agonist or } \\
\text { vehicle }\end{array}$ & $\begin{array}{l}\text { 12. Free } \\
\text { behavior } \\
\text { ( } 3 \text { days) }\end{array}$ & $\begin{array}{r}\text { 13. Seco } \\
\text { day with } \\
\text { balan } \\
\text { infustion } \\
\text { vehicle o } \\
\text { agon }\end{array}$ & $\begin{array}{l}\text { ad test } \\
\text { counter- } \\
\text { ced } \\
\text { f either } \\
\text { CB1R } \\
\text { ist }\end{array}$ & $\begin{array}{l}\text { Con } \\
\text { exp } \\
\text { and }\end{array}$ & $\begin{array}{l}14 . \\
\text { clusion of } \\
\text { eriments } \\
\text { histology }\end{array}$ \\
\hline
\end{tabular}

Fig 1 Schematic illustration of the experimental apparatus. a Effortbased T-maze decision-making task. The animals could choose between a low reward and a high-reward arm. However, they had to put in physical effort to climb a barrier in order to gain a higher reward. b Delay-based Tmaze decision-making task. The animals could choose between an immediately available small reward and a delayed large reward. c Sequence of training and experimental procedures for both tasks. Stages 5 , 7 , and 9 indicates testing days during the original differential cost tasks and stages 11 and 13 refer to testing days during control equal cost tasks 
$7 \mathrm{~min}$ in groups of three with ten food pellets (45 $\mathrm{mg}$ food reinforcement pellets, Formula A/I; P.J. Noyes) in the food wells in both goal arms.

Experiment 1 Following habituation to the maze, the animals (experiment 1a: $n=10$ and experiment $1 \mathrm{~b}: n=8$ ) were randomly assigned to receive high reward in the left arm or in the right arm. For the next 2 days, four pellets were placed in the highreward arm (HRA) and two pellets in the low-reward arm (LRA) for every animal, and the animals were placed individually in the start arm and were allowed to sample the pellets from both sides without a barrier in any of the goal arms. The same procedure was carried out for three additional days, but instead of one trial per day, the animals completed four trials per day. Subsequently, animals were allowed to sample food from only one of the goal arms. They performed two "forced" trials and ten "choice" trials per day for three further days. At this stage, the animals had free access to both HRA and LRA without any barrier on the way. During this phase, the animals had to choose one of the goal arms and they were removed from the maze following the consumption of the reward in the chosen arm well. Once the animals had reached an average high-reward choice (HRC) of $80 \%$ or more, a barrier of $5 \mathrm{~cm}$ high made of wire mesh was placed in the midpoint of the HRA to increase physical effort cost of the high reward. Similarly, after a few days of training in this phase and exceeding the average HRC of $80 \%$, the barrier height was increased in $5 \mathrm{~cm}$ steps till the maximum height of $30 \mathrm{~cm}$ at the end of training. The animals needed 2-4 days to complete each of these phases of the decision training, and a few more days of training were then conducted to consolidate the task before animals underwent the surgery for cannula implantation. One rat from group 1a was not willing to perform the task and follow the training and was thus excluded from the experiment.

Experiment 2 A separate group of animals (experiment 2a: $n=10$ and experiment $2 \mathrm{~b}: n=8$ ) were used for this experiment. Following habituation to the maze, animals were randomly assigned to receive high rewards in the left arm or in the right arm. For the next 2 days, ten pellets were placed in HRA and two pellets in the LRA for every animal and the animals were placed individually in the start arm and were allowed to sample the pellets from both sides with all four doors in an open state. The same procedure was carried out for three additional days, but instead of one trial per day, the animals completed four trials per day. Subsequently, animals were allowed to sample food from only one of the goal arms. They performed two "forced" trials and ten "choice" trials per day for three further days. At this stage, door B was closed in both goal arms and once the rat entered a goal arm, door A was closed and door B was opened and the animal had access to the chosen food well. During this phase, the animals had to choose one of the goal arms and they received an immediate reward in both arms. Once the animals had reached an average HRC of $80 \%$ or more, a delay of $5 \mathrm{~s}$ was introduced to the HRA. Similarly, after a few days of training in this phase and exceeding the average HRC of $80 \%$, the delay was increased to 10 and then $15 \mathrm{~s}$. One rat was not able to perform the task and follow the training and was excluded from the experiment. The animals needed 4-6 days to complete each of these phases of the decision training, and a few more days of training were then conducted to consolidate the task before animals underwent the surgery for cannula implantation.

\section{Surgery}

After an induction of anesthesia, achieved using a mixture of ketamine $(100 \mathrm{mg} / \mathrm{kg})$ and xylazine $(8 \mathrm{mg} / \mathrm{kg})$ intraperitoneal (i.p.) injection, rats were immobilized in a stereotaxic device (Stoelting Co., USA). An incision was made along the midline on the head of the rats and the skull was exposed. Stainless steel, 23 gauge guide cannulae were implanted bilaterally $1 \mathrm{~mm}$ above the target locations. The coordinates for target locations (ACC and OFC) were determined from a rat brain atlas (Paxinos and Watson 2007) as follows: $1.2 \mathrm{~mm}$ anterior to bregma, $0.8 \mathrm{~mm}$ lateral to the midline, and $2 \mathrm{~mm}$ ventral to the skull for ACC and $3.5 \mathrm{~mm}$ anterior to bregma, $2.3 \mathrm{~mm}$ lateral to the midline, and $5.4 \mathrm{~mm}$ ventral to the skull for OFC. After securing the guide cannulae in place, dental acrylic cement (Paladur) was applied to fix the implants. Before recovery from the anesthesia, the animals received $0.05 \mathrm{mg} / \mathrm{kg}$ buprenorphine and baytril (ciprofloxacin). Both drugs were administered s.c. and buprenorphine was repeated after $12 \mathrm{~h}$. Removable stylets with the same length as the guide cannulae were inserted into the guide cannulae and were only removed during microinjections. Following the surgery, rats were housed individually, were monitored on a daily basis, and were allowed to recover from the surgery at least for 10 days before restarting any behavioral training. For 2 days before the surgery and during the recovery period, rats had free access to food and water.

\section{Microinjections and behavioral testing}

Experiment 1 a Following recovery from the surgery and reestablishment of the restricted food regime, the animals completed two "forced decision" trials followed by ten "choice" trials of the effort-based T-maze decision-making task per day for 2-3 days, until they reached the presurgery performance rate. To ensure steady behavior, the training was continued for 10 days. ACC injections were counterbalanced such that animals first received either potent cannabinoid agonist (ACEA) or dimethyl-sulfoxide (DMSO; vehicle). During the test days, after the "forced decision" trials, the animals received bilateral microinjection of either ACEA (Tocris 
Bioscience UK, $0.73 \mu \mathrm{g} /$ side dissolved in DMSO) or vehicle in the ACC in a counterbalanced manner; the animals that received local ACEA treatment on the first test day were subjected to local vehicle treatment and vice versa. The animals performed the task for 3 days without any manipulation between test days with microinjections. The dose we used falls within the dose range of previous studies employing intracerebral application of high-affinity cannabinoid agonists including ACEA and CP55940 (Fogaca et al. 2012; Miller et al. 1998; Moreira et al. 2007; Robbe et al. 2006). All microinjections were administered in a volume of $0.5 \mu \mathrm{l}$ per side over $60 \mathrm{~s}$ using a stainless steel needle (30 gauge) which was directly inserted into the guide cannula, protruding $1 \mathrm{~mm}$ beyond the tip of the cannula. The injector cannula was connected to a $1-\mu$ l Hamilton syringe by polyethylene tubing (PE-20). The injectors were left in place for $60 \mathrm{~s}$ after the injection to allow diffusion and were then replaced by the stylets. The behavioral testing was continued after the microinjections in exactly the same way as before and the animals' behavior was recorded. After the microinjection of ACEA and vehicle, the animals received a microinjection of cannabinoid antagonist, AM251 (Tocris Bioscience, 5.55 $\mathrm{gg} / \mathrm{side}$ dissolved in DMSO) to study the influence of CB1R inactivation of the ACC in the effort-based decision-making task. A 3-day interval of behavior without any manipulation was maintained for all microinjections.

After the completion of all behavioral testing with microinjection in the effort-based decision task, the animals were trained on a control task in which a similar $30 \mathrm{~cm}$ height barrier was newly introduced also to the LRA, to test possible involvement of spatial preference or memory in the decisions of the animals. The training continued for about 10 days to ensure that the changed rule was well established for the animals. On the test days, the animals received microinjections of ACEA and saline in a counterbalanced manner and behavioral parameters were analyzed as above.

Experiment $1 \mathrm{~b}$ The experimental procedure and treatment schedule was performed exactly in the same way as in the differential effort task in experiment 1a. The only difference was however, that, the rats in this group had cannulae in the OFC and received local treatments in the OFC.

Experiment $2 a$ Following recovery from the surgery and reestablishment of the restricted food regime, the animals completed two "forced decision" trials followed by ten "choice" trials of the delay-based T-maze decision-making task per day for 2-3 days, until they reached the presurgery performance rate. To ensure steady behavior, the training was continued for 10 days. One rat did not recover and was not able to follow the task as he did before the surgery and therefore was excluded from the experiment. During the test days, after the "forced decision" trials, the animals received bilateral microinjection of either ACEA (Tocris Bioscience, $0.73 \mu \mathrm{g}$ /side dissolved in $\mathrm{DMSO}$ ) or vehicle in the OFC in a counterbalanced manner; the animals that received local ACEA treatment on the first test day were subjected to local vehicle treatment and vice versa. In this experiment also, the animals performed the task for 3 days without any manipulation between test days with microinjections. All microinjections were administered in a volume of $0.5 \mu \mathrm{l}$ per side over $60 \mathrm{~s}$ using a $1-\mu \mathrm{l}$ Hamilton syringe. Sixty seconds after the injection, the injectors were removed and were replaced by the stylets. The behavioral testing was continued after the microinjections in the same way as before and the animals' behavior was recorded. The animals performed the task for 3 days without any manipulation between the microinjection days. After the microinjection of ACEA and vehicle, the animals received a microinjection of cannabinoid antagonist, AM251 (Tocris Bioscience, $5.55 \mu \mathrm{g} /$ side dissolved in DMSO) to study the influence of CB1R inactivation of the OFC in the delay-based decisionmaking task. A 3-day interval of behavior (decision task) without any manipulation was maintained for all microinjections.

After the completion of all behavioral testing with microinjection in the delay-based decision task, the animals were trained on a control task in which a similar $15 \mathrm{~s}$ delay was newly introduced also to the LRA, to test possible involvement of spatial preference or memory in the decisions of the animals. The training continued for about 10 days to ensure that the changed rule was well established for the animals. On the test days, the animals received microinjections of ACEA and saline in a counterbalanced manner and behavioral parameters were analyzed as above.

Experiment $2 b$ Similar to experiment $2 \mathrm{a}$, the rats performed delay-based decision task. All experimental procedure was similar to differential delay task in experiment $2 \mathrm{a}$ with the only difference that the rats in this group had cannulae implanted in the ACC and received the local microinjections in the ACC.

\section{Locomotor activity control experiment}

The equal cost control experiments were exactly the same as the test experiments except equating the costs so they control for all cost-benefit decision-making irrelevant factors such as memory, locomotor ability, or possible spatial preferences in the same setting as test experiments. However, it can be argued that changes in the activity level as a result of cannabinoid system activation can bias animals' choice in the presence of differential costs. A further control experiment was conducted to measure spontaneous locomotor activity of the rats' following cannabinoid system manipulation. Two separate groups of rats were implanted with cannulae aimed at the ACC $(n=6)$ or the OFC $(n=6)$. The coordinates and 
surgery procedure were the same as described above. In both ACC and OFC groups, the animals received microinjections of ACEA, vehicle, and AM251 in the same counterbalanced manner as described above and then their activity was recorded in an open field. In these experiments also a 3-day interval of no manipulation between microinjections was applied.

The open field test was used to address the spontaneous motor activity profile following cannabinoid system manipulation in the ACC and OFC. Ten minutes after microinjection, rats were placed in the center of an open field measuring $30 \mathrm{~cm} \times 30 \mathrm{~cm}$ with $30 \mathrm{~cm}$ walls and were allowed to freely move inside the arena for $10 \mathrm{~min}$. The activity was recorded through a 3CCD camera (Panasonic Inc., Japan) mounted $2 \mathrm{~m}$ above the open field and was analyzed offline using Ethovision video tracking software (version 3.1, Noldus Information Technology, The Netherlands).

Tissue preparation, immunohistochemistry, and fluorescence microscopy

A separate group of six naïve male Wistar rats aging 3 months old were euthanized by i.p. injection of pentobarbital and were transcardially perfused with a saline $0.9 \%$ solution followed by a fixative containing $4 \%$ paraformaldehyde (PFA) in $0.1 \mathrm{M}$ phosphate-buffered saline solution (PBS). Whole brains were removed and stored in fresh PFA $4 \%$ solution at $4{ }^{\circ} \mathrm{C}$ overnight and then passaged to a solution of $30 \%$ sucrose in 0.1 M PBS until the tissues sank. The brains were then coronally cut into $50 \mu \mathrm{m}$ sections using a freezing microtome (Leica Biosystems) and serially collected sections were kept in $0.1 \mathrm{M}$ PBS to be immunohistochemically processed using the free-floating method. The slices were washed in PBS three times and then were incubated for $1 \mathrm{~h}$ in a PBS solution containing $5 \%$ bovine serum albumin and $0.5 \%$ TritonX100 to block unspecific reactions. The brain sections were then incubated for $24 \mathrm{~h}$ at room temperature in single primary antibodies or in a cocktail of two primary antibodies raised in different species diluted in the blocking solution. Primary antibodies that we used in our immunohistochemistry (IHC) experiments are as follows: guinea pig anti-CB1R C-terminus polyclonal antibody (1:1,000 dilution; Frontier Institute, Japan), rabbit anti-glial fibrillary acidic protein (GFAP) polyclonal antibody (1:300 dilution; DAKO, Denmark), rabbit anti-vesicular GABA transporter (VGAT) polyclonal antibody (1:100 dilution; Millipore, MA, USA), rabbit anti-tyrosine hydroxylase $(\mathrm{TH})$ polyclonal antibody (1:1,000 dilution; Millipore), rabbit anti-vesicular glutamate transporter type 1 (VGlut1) polyclonal antibody (1:300 dilution; Abcam, UK) and rabbit anti-serotonin transporter (SERT) polyclonal antibody (1:500 dilution; Abcam). Brain slices including the ACC and OFC were incubated in a blocking PBS solution containing one of the primary antibodies or a mixture of one of the every other primary antibody with anti-CB1R antibody. The following day, the sections were washed again in PBS three times and were incubated for $2 \mathrm{~h}$ in the blocking solution containing appropriate secondary antibody(ies). Alexa Fluor 568 goat anti-guinea pig $\operatorname{IgG}(\mathrm{H}+\mathrm{L} ; 1: 400$ dilution; Invitrogen, Life Technologies Co., CA, USA) and Alexa Fluor 488 donkey anti-rabbit $\operatorname{IgG}(\mathrm{H}+\mathrm{L} ; 1: 400$ dilution; Invitrogen, Life Technologies Co.) were the secondary antibodies used against primary antibodies in these experiments. The sections were then rinsed, mounted in an aqueous mounting media on gelatin-coated slides, and cover-slipped. In every staining run, there were negative controls in which the primary antibody was omitted. The 3D fluorescence images were acquired using confocal fluorescence microscopy with a Zeiss Axio Observer Z1 microscope with an ApoTome.2 (Carl Zeiss, Germany) attachment and structured illumination technique. $Z$ serial sections were acquired at the optimal Nyquist resolution for each objective, i.e., at $0.54 \mu \mathrm{m}$ with the $\times 20$ objective ( 0.8 aperture) and at $0.27 \mu \mathrm{m}$ with the $\times 63$ objective (1.4 aperture) (Webb and Dorey 1995). Z projection from stack to single image, color levels adjustment, scale bar inclusion, and RGB conversion was performed using appropriate FIJI plug-ins (Schindelin et al. 2012).

\section{Histology}

After the completion of all tests, the animals were deeply anesthetized by i.p. injection of pentobarbital and transcardially perfused with saline $0.9 \%$ and PFA $4 \%$. Whole brains were removed and were fixed in PFA $4 \%$ overnight and then in sucrose $30 \%$ until they sank. The brains were cut into $50-\mu \mathrm{m}$ sections in the coronal plane using a freezing microtome (Leica Biosystems) and underwent Nissl staining. Injection sites for available histological placements $(n=9,8,5$, 4 for main experiments $1 \mathrm{a}, 2 \mathrm{a}$ and control experiments $1 \mathrm{~b}$ and $2 \mathrm{~b}$ respectively) are depicted in Fig. 2.

\section{Results}

Effects of cannabinoid system manipulation in ACC on effort-based decision making

In experiment 1 , rats performed a simple cost-benefit T-maze decision-making task involving effort cost. Two goal arms differed in the amount of available reward and in the magnitude of physical effort the rats incurred to gain rewards. The animals were extensively trained before surgery and rapidly established presurgery performance (i.e., high-reward choices or HRCs) following a recovery from cannulae implantation surgery. After several days of consistent behavior, the animals were randomly assigned to receive intra-ACC (experiment 1a) microinjection of the potent $\mathrm{CB} 1 \mathrm{R}$ agonist ACEA, or vehicle 
a

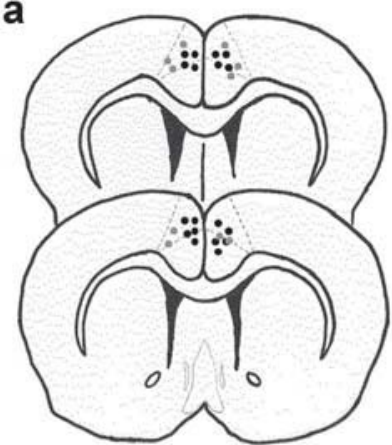

b

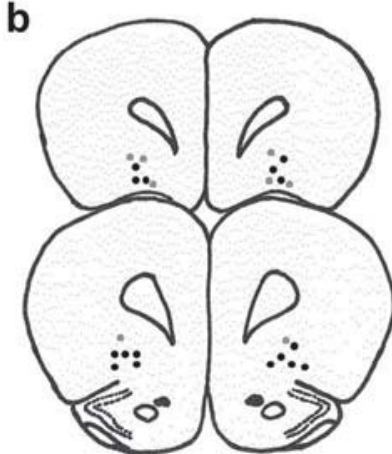

Bregma

1.6

1.2

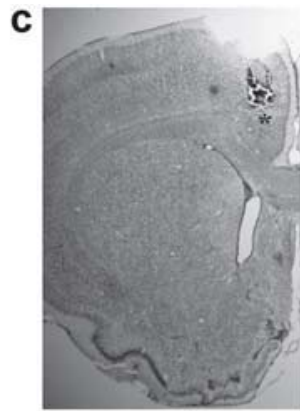

4

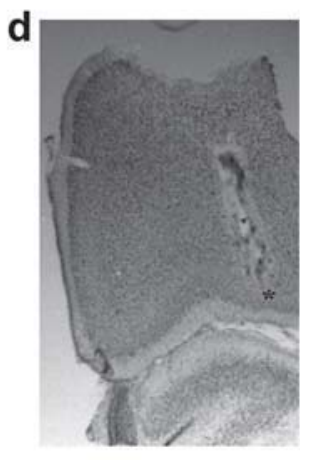

Fig 2 Anatomical reconstruction approximate bilateral microinjection sites in a the ACC $(n=9)$ and $\mathbf{b}$ the OFC $(n=8)$. Each "•" or "•" symbol represents an injection site for an individual animal that was used in the main experiments or area control experiments, respectively. The distance of every coronal plane anterior to bregma is denoted on the right side of the reconstruction schematics. Coronal Nissl-stained sections showing cannula tracks and injection sites, marked with an "**" symbol in $\mathbf{c}$ the $\mathrm{ACC}$ and $\mathbf{d}$ the $\mathrm{OFC}$

in a counterbalanced manner. Subsequently, after a 3-day interval of behavior without any manipulation, all the animals received a cannabinoid antagonist, AM251. Figure 3 (left panel) shows the mean percentage of HRCs obtained for vehicle, AM251, and ACEA microinjection groups. A repeated measures ANOVA showed a main effect of the local ACC treatment $\left(F_{2,16}=179.9 ; p<0.0001\right)$ on the performance of the rats. While vehicle-microinjected rats continued to choose high-reward/high-effort arm, cannabinoid agonist microinjected rats exhibited a strong reduction in HRCs, which was significantly different than vehicle-microinjected (Bonferroni post hoc analysis, $p<0.0001$ ) or cannabinoid antagonist-microinjected (Bonferroni post hoc analysis, $p<0.0001)$ rats. Thus, the activation of CB1R in the ACC shifted the preference of animals from deciding to expend physical effort to gain higher reward to choosing a small reward at low physical cost. AM251 microinjection into the ACC in our experimental setting could not address whether cannabinoid system inactivation in the ACC has opposite effects on effort cost processing due to the possible ceiling effect. This was the basis to exclude AM251 microinjection in the subsequent control task.

To evaluate whether the reduction in the HRCs as a result of intra-ACC microinjection of ACEA specifically affects effort-based decision making, rather than having a more general impact on spatial preference or memory, we proceeded to train the same group of animals on an additional control task. In this task, the differential rewards were maintained as before (four vs. two pellets) but the physical effort cost the rats incurred to gain different-sized rewards was set to the same value for both sides (30 cm barrier). Fig. 3 (middle panel) shows mean percentage of the rats' HRCs in this new "equal effort" control task. A two-way repeated measures ANOVA on ACEA and vehicle microinjection results in both tasks revealed a significant main effect of the treatment $\left(F_{1,8}=\right.$ 202.1; $p<0.0001)$ and a significant main effect of the task $\left(F_{1,8}=153.6 ; p<0.0001\right.$; "differential effort" vs. "equal effort" control task) and a significant treatment $\times$ task interaction $\left(F_{1}\right.$, $\left.{ }_{8}=169.7 ; p<0.0001\right)$. A subsequent Bonferroni post hoc analysis revealed a significant reduction in the rats' HRCs with ACEA compared to vehicle in the original task $(p<0.0001)$ but not in the "equal effort" control task. Along similar lines, there was a significant difference in HRCs between the original and the "equal effort" control task in the ACEA $(p<0.0001$; Bonferroni post hoc test) but not the vehicle treatment condition.

To assess whether the reduction in the HRCs as a result of local cannabinoid system activation during effort-based decision task is specific to the ACC, a separate group of rats (experiment $1 \mathrm{~b}$ ) were trained and implanted with cannulae in the OFC. Figure 3 (right panel) shows the HRCs of the rats following counterbalanced intra-OFC microinjection of cannabinoid agonist and vehicle. Cannabinoid agonist or vehicle injection in the OFC had no effect on the HRCs as confirmed by a repeated measures ANOVA $(p>0.1)$. A separate two-way ANOVA with repeated measures in one factor (treatment) compared the effects of the treatment and microinjection area and showed a significant main effect of the treatment $\left(F_{1,15}=\right.$ $151.3 ; p<0.0001)$, a significant main effect of brain area $\left(F_{1}\right.$, $\left.{ }_{15}=75.2 ; p<0.0001\right)$ and a significant interaction $\left(F_{1,15}=\right.$ $116.5 ; p<0.0001)$. Taken together, the behavioral changes in effort-based decision making were specific for the ACC but not OFC cannabinoid activation and these effects were specific to the original decision task with differential efforts, and were not observed during a control task with equal effort costs, ruling out reward memory or spatial preference confounds.

\section{Effects of cannabinoid system manipulation in OFC} on delay-based decision making

In experiment 2, rats performed a cost-benefit T-maze decision-making task involving delay cost. As in experiment 1, the two goal arms were different in the amount of available reward and the magnitude of the cost the rats incurred to gain 


\section{Effort-Based Decision Making}

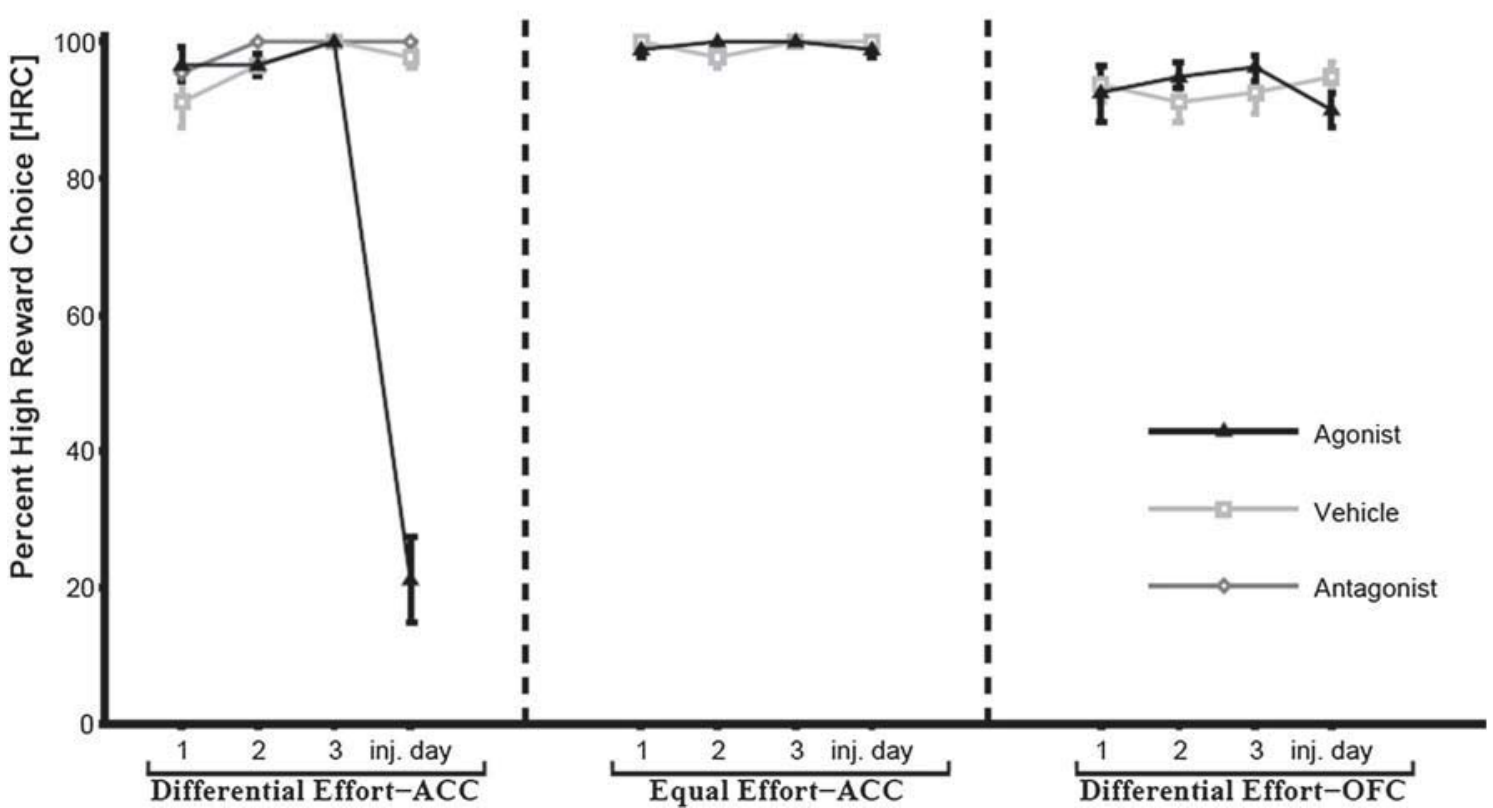

Fig $3 \mathrm{CB} 1 \mathrm{R}$ Agonist application in $\mathrm{ACC}$ increases effort averseness during decision making. Mean percentage of high-reward choices (HRCs, $\pm \mathrm{SEM})$ on the test days, where rats received different local treatments in the ACC (left and middle panel) and OFC (right panel) and during 3 days preceding the test days without any manipulation. The left panel shows the performance on the original differential effort decision task. The

these rewards. However, while in experiment 1, the costs were in terms of physical effort, in experiment 2 , the rats based their decision on different delay costs to achieve different-sized rewards. Similar to experiment 1, the animals established high ratio of HRCs following a postsurgery recovery and exhibited a consistent behavior for several days. Subsequently, the rats were randomly assigned to receive a local administration of either ACEA or vehicle in the OFC (experiment 2a) in a counterbalanced manner. After a 3-day interval of behavior without any manipulation, all the animals received a cannabinoid antagonist, AM251. The mean percentages of HRCs in delay-based decision task in vehicle, AM251, and ACEA groups are displayed in Fig. 4 (left panel). A repeated measures ANOVA showed a significant main effect of the local OFC treatment $\left(F_{2,14}=2,076 ; p<0.0001\right)$. Intra-OFC microinjection of cannabinoid agonist led to a drastic decline in the preference of the rats for delayed large rewards being significantly different than vehicle-microinjected (Bonferroni post hoc test, $p<0.0001$ ) or cannabinoid antagonist-microinjected (Bonferroni post hoc test, $p<0.0001$ ) rats. Thus, as a result of $\mathrm{CB} 1 \mathrm{R}$ activation in the $\mathrm{OFC}$, the rats exhibited an impulsive pattern of decision making switching from deciding to wait to gain a large reward to choosing to receive small but immediate reward. Similar to the effort-based decision task, the preference of the rats to high-cost/high-reward choice following AM251 microinjection into the OFC was not different than middle panel shows the performance on a control task in which the same group of rats had to expend an equal amount of physical effort for both choice options. The CB1R antagonist was not tested in the equal effort task. The right panel shows the performance on the original differential effort task in a separate group of rats receiving local treatments in the OFC. inj. day: injection day (i.e., test day)

vehicle treatment, which we attribute to a ceiling effect resulting from the high $\mathrm{HRC}$ percentage in the control condition.

The same group of rats was subsequently trained on a second task in which the decision was simply based on differential reward but equal cost by introducing an equal 15-s delay for the LRA. Rats rapidly learned the new task. IntraOFC microinjection of vehicle or ACEA had no effect on the mean percentages of HRCs, as shown in Fig. 4 (middle panel). A two-way repeated measures ANOVA revealed a significant main effect of the treatment $\left(F_{1,7}=2,767 ; p<0.0001\right)$ and a significant main effect of the task $\left(F_{1,7}=794.3 ; p<0.0001\right.$; "differential delay" vs. "equal delay" control task) and a significant treatment by task interaction $\left(F_{1,7}=1,270\right.$; $p<0.0001)$. A subsequent Bonferroni post hoc analysis revealed a significant reduction in the rats' HRCs with ACEA compared to vehicle in the original task $(p<0.0001)$ but not in the "equal delay" control task. Along similar lines, there was a significant difference in HRCs between the original and the "equal delay" control task in the ACEA $(p<0.0001$; Bonferroni post hoc test) but not the vehicle treatment condition.

A separate group of rats (experiment $2 b$ ), trained and implanted with cannulae in the ACC, was then used to test whether the cannabinoid agonist induced decline in the HRCs during delay-based decision task is specific for the OFC. As 
shown in Fig. 4 (right panel), cannabinoid agonist or vehicle microinjection in the ACC had no effect on the HRCs $(p>0.1)$. A two-way ANOVA with repeated measures in one factor (treatment) compared the effects of the treatment and injection area on HRCs during delay-based decision task and showed a significant main effect of the treatment $\left(F_{1,14}=\right.$ 511.6; $p<0.0001)$, a significant main effect of area $\left(F_{1,14}=\right.$ $249.1 ; p<0.0001)$, and a significant interaction $\left(F_{1,14}=416.6\right.$; $p<0.0001)$. This demonstrates that the behavioral changes in delay-based decision making were specific for cannabinoid receptor 1 activation in the OFC but not the ACC and that these effects were not the result of reward memory or spatial preference biases confounds.

Effects of cannabinoid system manipulation in ACC and OFC on locomotor activity

A potential concern is that the changes in decision patterns following microinjection of $\mathrm{CB} 1 \mathrm{R}$ agonist into the ACC and OFC can result from the changes in locomotor activity, such that cannabinoid system activation in ACC and OFC might lead to general hypoactivity and hyperactivity, respectively, which in turn makes rats to appear to choose apathetically or impulsively. To rule out this potentially confounding factor, we conducted a control experiment measuring spontaneous locomotor activity. Following microinjection of vehicle, AM251, or ACEA, rats were placed in an open field and were allowed to explore for $10 \mathrm{~min}$. The open-field task was conducted to measure general activity levels of the rats as a function of manipulation of cannabinoid system in the ACC or OFC. The profile of spontaneous locomotor activity following different treatments in the two brain areas was highly similar, suggesting that locomotor activity per se was largely unaffected by cannabinoid system manipulations in the OFC and ACC, as shown in Fig. 5. This was confirmed by two separate two-way repeated measures ANOVA for each brain region with treatment condition and time bin as factors $(p>0.1)$.

Distribution and localization of CB1Rs in the ACC and OFC

It is well established that CB1Rs are localized at GABAergic axonal ends in many brain regions (Bodor et al. 2005; Kawamura et al. 2006; Matyas et al. 2006) including PFC (Chiu et al. 2010). There are also reports demonstrating CB1Rs localization on glutamatergic axonal ends (Domenici et al. 2006; Kawamura et al. 2006; Reguero et al. 2011) and on astrocytes (Navarrete and Araque 2008) in various brain regions. We thus examined the pattern of distribution of $\mathrm{CB} 1$ receptors and colocalization of these receptors with major neuronal populations including GABAergic, glutamatergic, dopaminergic, and serotoninergic neurons and astrocytes in the ACC and OFC. In both ACC and OFC, there was an intense staining of CB1 receptors in fibrous, apparently woven

\section{Delay-Based Decision Making}

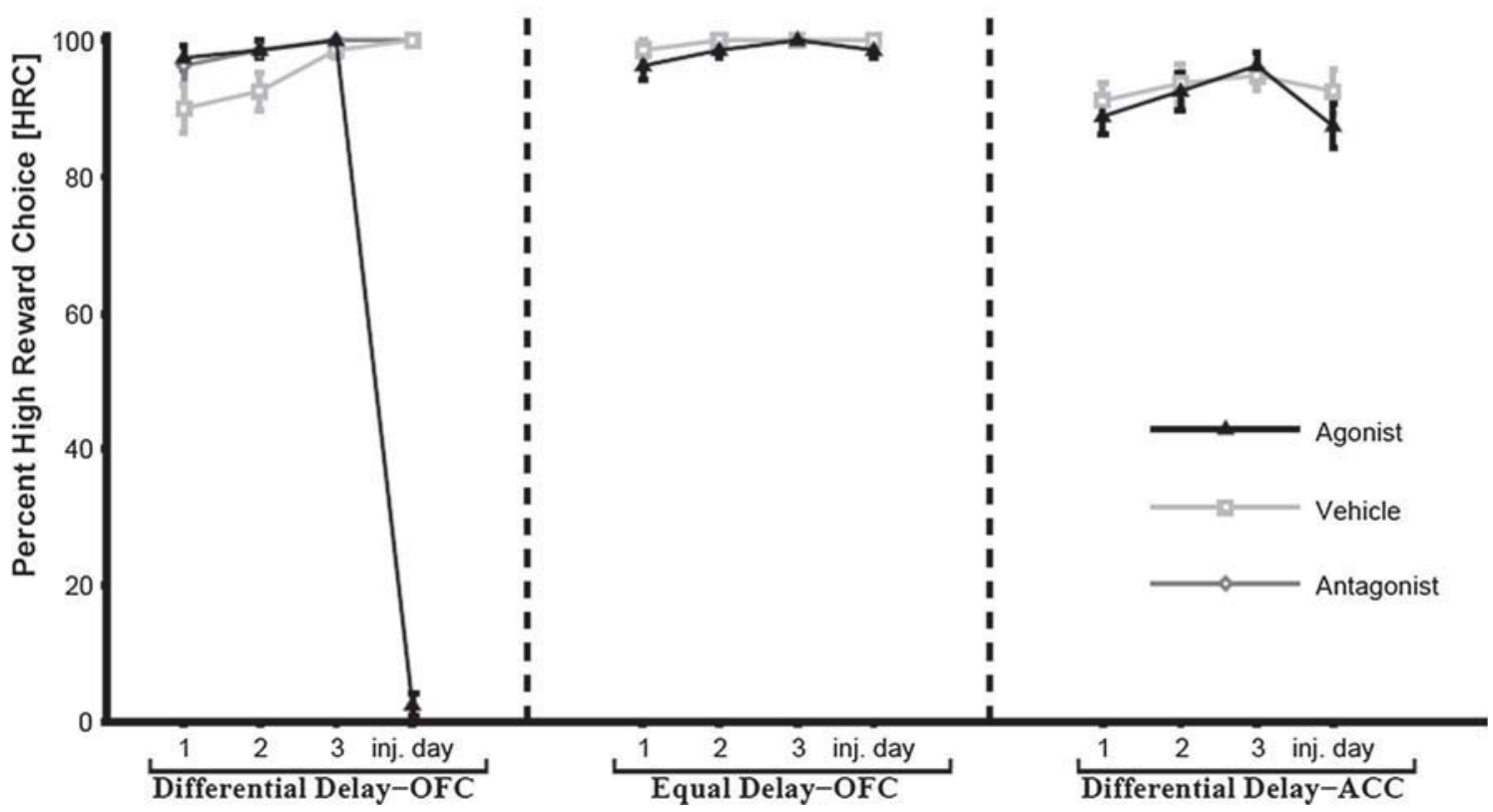

Fig $4 \mathrm{CB} 1 \mathrm{R}$ application in the OFC increases delay averseness during decision making. Mean percentage of HRCs $( \pm$ SEM) of the rats in the differential delay decision task (left panel) and a control task using equal delays (middle panel). On the test days rats received local administration of different treatments in the OFC; decision performance is also shown for the three preceding days without any pharmacological manipulation. The right panel shows performance of a separate group of rats in differential delay task. These rats received local treatments in the ACC. inj. day: injection day (i.e., test day) 


\section{Spontaneous locomotor activity}
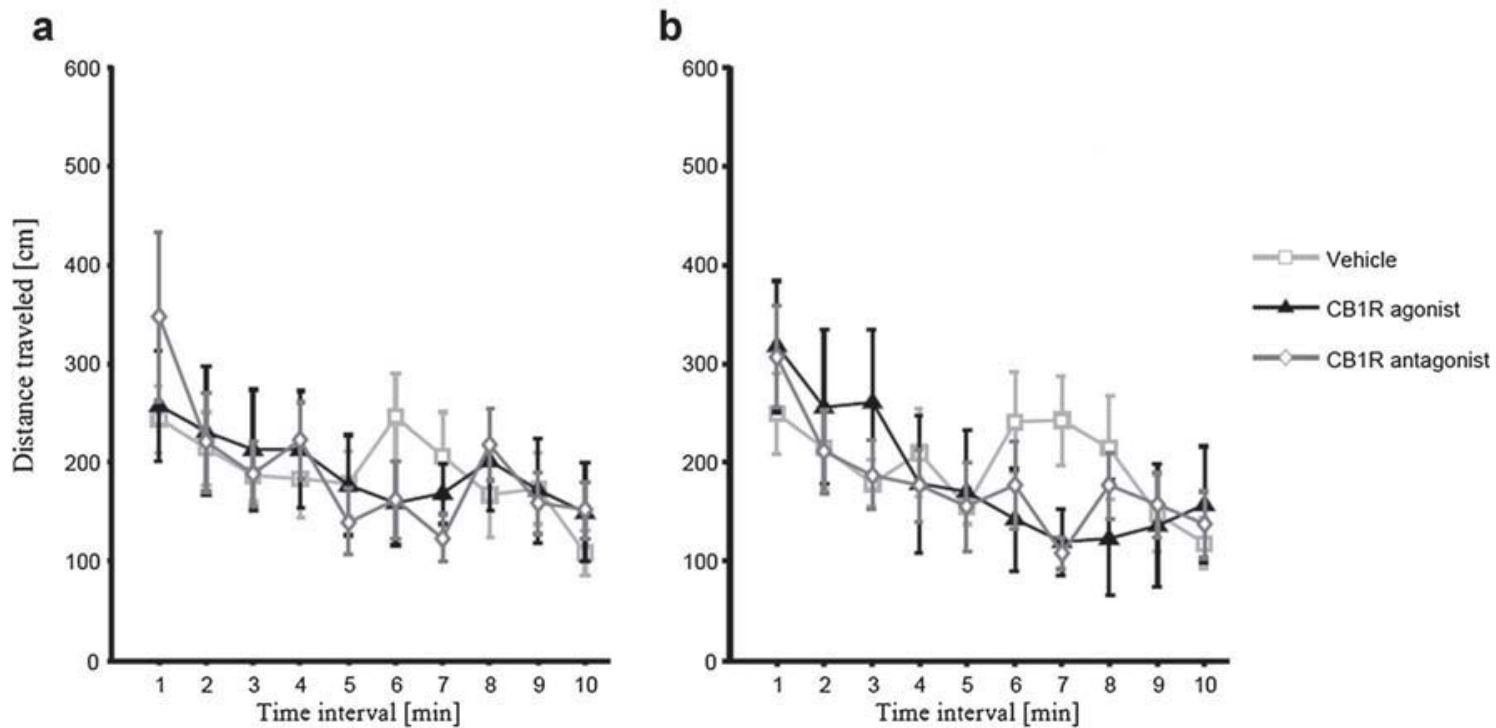

Fig 5 Spontaneous locomotor activity is unaffected by cannabinoid treatments spontaneous locomotor activity during exploration (mean \pm SEM) following different treatments in a the ACC and $\mathbf{b}$ the OFC during 1-min time intervals for a 10-min period measured in an open field

forms with almost no distinguishable staining on cell bodies. Figures 6 and 7 show the distribution and colocalization patterns of CB1Rs in the ACC and OFC, respectively. In both regions, there was widespread colocalization of $\mathrm{CB} 1 \mathrm{Rs}$ with GABAergic axonal ends but only relatively few colocalizations were apparent with dopaminergic, serotonergic, and glutamatergic axons. We did not observe any colocalization with astrocytes in either area.

\section{Discussion}

Our results demonstrate profound effects of cannabinoid system activation on frontal brain circuits of decision making. Cannabinoid agonist application modified decision behavior, such that animals tended to make suboptimal decisions by avoiding decision costs. In rats performing an effort-based decision task, animals nearly always choose to expend additional physical effort to obtain a high reward. Microinjection of the potent $\mathrm{CB} 1 \mathrm{R}$ agonist into the $\mathrm{ACC}$, but not the $\mathrm{OFC}$, in an effort-based decision task led to a large decrease in HRCs. Similarly, microinjection of a CB1R agonist into the OFC, but not the ACC, in rats performing a delay-based decision task dramatically increased choice of a low but immediately available reward. This shows a double dissociable pattern of cannabinoid effects in the ACC and OFC during effort-based and delay-based decision making, respectively. In the absence of a need to integrate costs and benefits during decision making, rats chose high-reward arms in both tasks irrespective of cannabinoid system activation. An additional, control experiment showed that the spontaneous locomotor activity was not affected by any of the treatments, suggesting that simple motor hypo- or hyperactivity cannot account for the observed changes in decision-making behavior. The cannabinoid system manipulations we performed produced decline in rats HRCs that were similar in size to those produced by lesions in the ACC and OFC (Rudebeck et al. 2006). This suggests that local cannabinoid system activation may play a crucial role in modifying ACC and OFC decision circuits.

The involvement of the ACC in several dimensions of reinforcement-guided decision making is generally well acknowledged (Rushworth et al. 2007). Lesion and single neuron studies have shown that the ACC plays a critical role in decisions that require putting in a certain amount of effort to gain a better reward (Kennerley et al. 2011; Rudebeck et al. 2006). Similarly, several lines of evidence have shown that while the OFC is also a critical region in decision making, it has a dissociable role than the ACC in many respects including cost processing, i.e., delay costs in the OFC vs. effort costs in the ACC (Rushworth et al. 2007). The ACC and OFC have connections with several other brain regions including basolateral amygdala (BLA) and nucleus accumbens (NAc) (Rushworth et al. 2007) and the input from the ACC and OFC to NAc is integrated with input from other regions (Grace 2000; Grace et al. 2007). Both BLA and NAc are involved in the processing of cost-benefit decision making (Rushworth et al. 2007). Our results provide the first direct evidence linking the cannabinoid system to frontal cortical areas during cost-benefit decision making: In the ACC, cannabinoid 
Fig 6 CB1R distribution and colocalization in ACC distribution of $\mathrm{CB} 1$ receptors and VGAT, VGlut1, SERT positive terminals and $\mathrm{TH}$ positive neurons and GFAP positive astrocytes in the ACC. Coronal $50 \mu \mathrm{m}$ sections were immunostained for CB1R in red and for all other markers in green. a There is a dense CB1R expression in the ACC (b) No CB1R localization was found on GFAP positive astrocytes. Only few terminals show CB1R colocalization with (c) SERT or (d) $\mathrm{TH}$ positive axons and are marked with white arrowheads. $\mathbf{e}$ In contrast, a high rate of CB1R colocalization with VGAT was observed. Arrowheads show only some of the colocalizations. Apart from some evident colocalization in axosomatic synapses to pyramidal neurons, whose bodies are well delimitated, some of the colocalizations are not related to pyramidal cell bodies. f Only few $\mathrm{CB} 1 \mathrm{R}$ colocalizations were found with VGlut1 positive terminals. Magnifications: $\times 20$ for $\mathbf{a}, \times 63$ for b-f. Scale bars: a, $50 \mu \mathrm{m}$; b-f, $10 \mu \mathrm{m}$
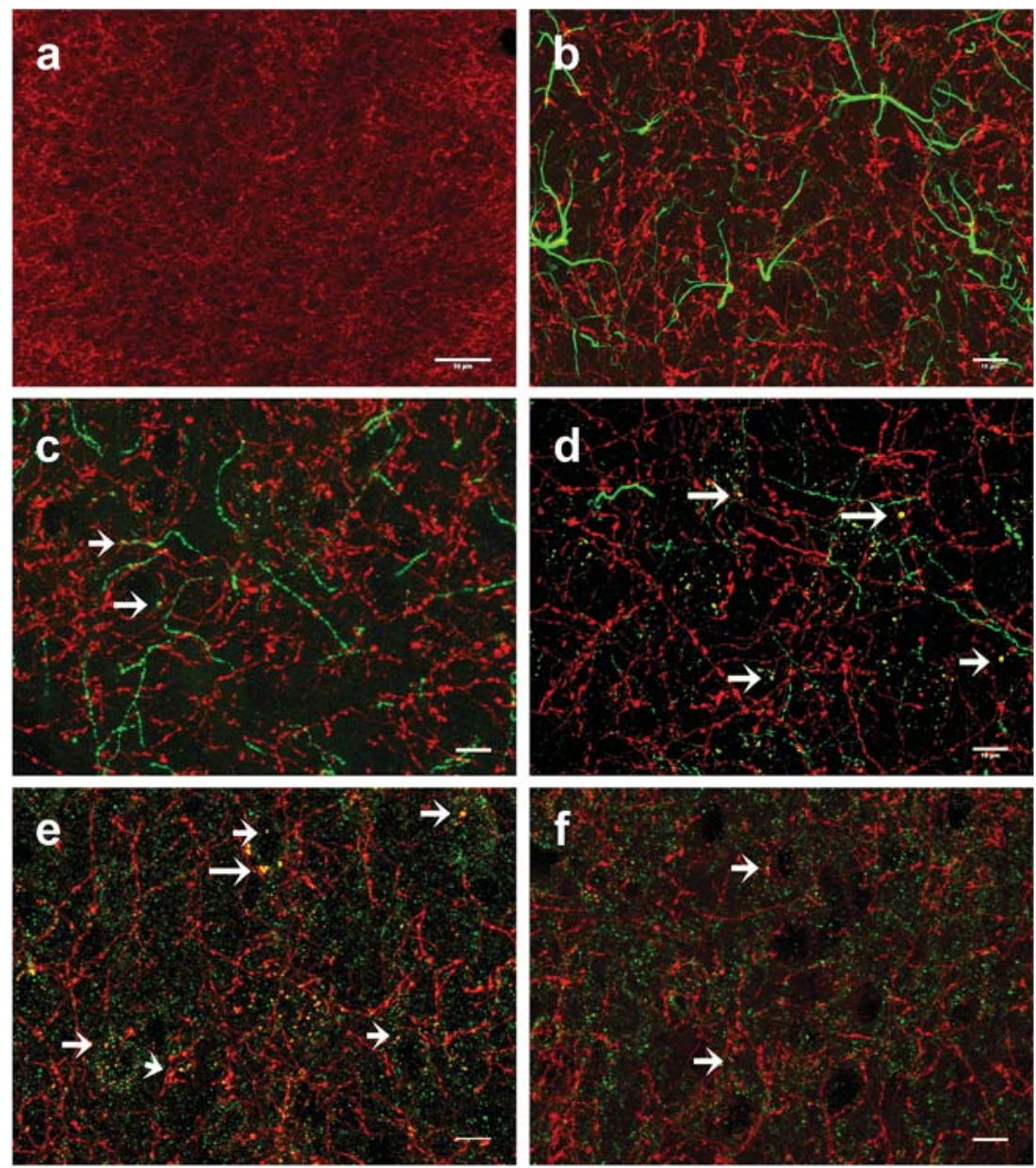

activation biases rats' decisions from putting in physical effort to attain a higher reward to preferring a small reward at a lower physical effort cost and in the OFC it shifts rats' decisions away from waiting for a large reward toward an immediately available small reward. Previous work has shown that the effects of cannabinoids on impulsive decision making have been complex and controversial. Some studies demonstrated increased impulsive choice (Hernandez et al. 2014; Loflin et al. 2014) while others showed unaffected or decreased impulsive choice following cannabinoid administration (McDonald et al. 2003; Pattij et al. 2007; Wiskerke et al. 2011). It has also been suggested that genetic factors influence the effects of cannabinoids on impulsive decision making (Boomhower et al. 2013) and polymorphisms in CB1R gene is involved in differential trait impulsivity (Ehlers et al. 2007). On effort-based decision making, one study (Sink et al. 2008) showed that $\mathrm{CB} 1 \mathrm{R}$ blockade decreased the rats willingness to press a lever to acquire preferred food and another study suggested that coadministration of a cannabinoid agonist with "ecstasy" did not change deficits during effort-based decision making induced by the administration of "ecstasy" alone (Schulz et al. 2013). While these studies implicate cannabinoid system in the processing of cost-benefit decision making, they have generally used systemic administration of cannabinoid agents. It follows that the net behavioral effect is the result of cannabinoid action in several brain regions including not only the ACC and OFC but also the VTA, nucleus accumbens, and other brain regions expressing CB1Rs. By contrast, our study employed local administration, allowing us to clarify specifically the role of cannabinoid action in the ACC and OFC during decision making. Taken together, our findings suggest that the cannabinoid system may have opposite effects on different components of the neural circuitry underlying decision making.

Among neurotransmitter systems, the involvement of dopamine (DA) system in effort-based decision making has been well established, particularly for the mesolimbic dopaminergic projection system that innervates the NAc (Hauber and Sommer 2009; Mai et al. 2012; Salamone et al. 1994). The involvement of the mesocortical dopaminergic pathway projecting to the ACC in decision making is less clear and remains a subject of debate. For example, while one study has failed to find evidence for a role of dopaminergic projections from the ventral tegmental area (VTA) to the ACC in effortbased decision making (Walton et al. 2005), other authors have demonstrated that blockade or downregulation of 
Fig 7 CB1R distribution and colocalization in OFC Confocal images showing (a) distribution of $\mathrm{CB} 1$ receptors (red) and double immunofluorescence for CB1R with (b) GFAP positive astrocytes, (c) SERT positive terminals, and (d) $\mathrm{TH}$ positive neurons, (e) VGAT, and (f)

VGlut1 positive terminals. CB1R is immunostained in red and all other markers in green. Double confocal microscopy show few CB1R colocalization with SERTpositive terminals, $\mathrm{TH}$ positive neurons, and VGlut1 positive terminals but no colocalization with GFAP positive astrocytes and considerable colocalizations with VGAT. Magnifications: $\times 20$ for a, $\times 63$ for b-f. Scale bars: a, $50 \mu \mathrm{m}$; b-f, $10 \mu \mathrm{m}$
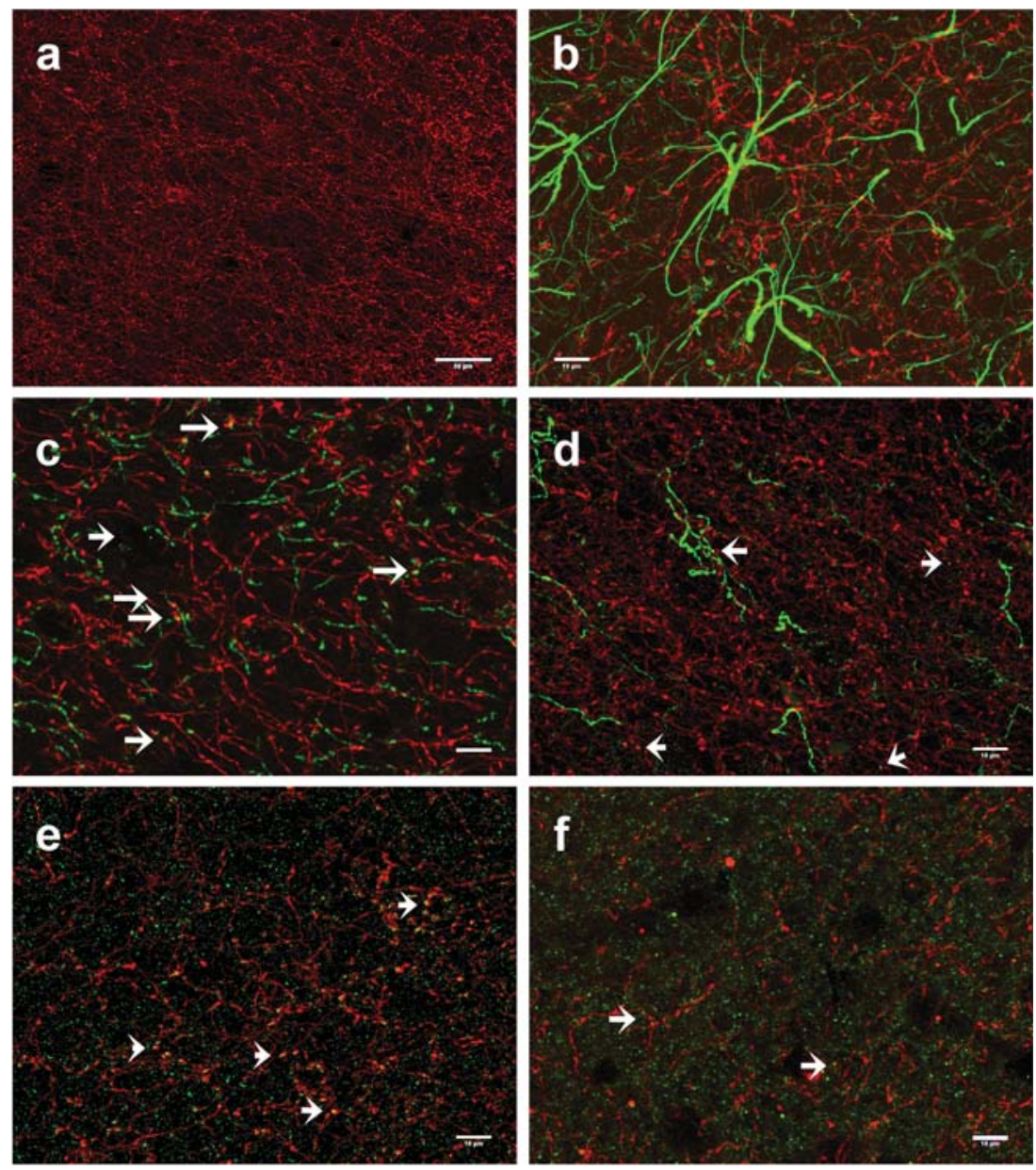

dopaminergic neurotransmission in the ACC makes animals effort aversive during decision making (Schweimer and Hauber 2005; 2006). As the latter authors discussed, the differences in the level of DA depletion may largely account for this discrepancy between results. A substantial DA depletion or blockade of D1 receptors in the ACC is necessary to impair effort-based decision making. It has also been demonstrated that DA system in the OFC plays a role in delay-based decision making (Zeeb et al. 2010). Blockade of dopamine receptors in the OFC decreased the number of large delayed reward choices. In our study, we have demonstrated that there are $\mathrm{CB} 1 \mathrm{R}$ colocalizations on DA neurons in the ACC (Fig. 6d) and OFC (Fig. 7d). Although these colocalizations were overall relatively infrequent, they may be present on a notable proportion of DA afferents, since these are relatively dispersed. This suggests that the CB1R-related cost averseness during decision making may at least in part be mediated by cannabinoid suppression of DA neurotransmission, although the overall relatively weak colocalization suggests that this is probably not the main mechanism of cannabinoid influence on decision making in the ACC. Our IHC experiments have also demonstrated a similar sparse pattern of CB1R colocalizations on serotonergic neuronal ends. To our knowledge, serotonergic neurotransmission is not implicated in effort-based decision making while the involvement of serotonergic system in the delay-based decision making is well established (Denk et al. 2005; Hadamitzky et al. 2009). This suggests that modulation of serotonergic projections to the OFC might partly contribute to cannabinoid-induced delay averseness during delay-based decision making.

Of the neuronal populations tested, GABAergic axonal ends exhibited the strongest colocalization with CB1Rs in our immunohistofluorescence experiments. This observation is in agreement with the notion that predominant effects of CB1Rs activation in the prefrontal cortex in adulthood are mediated through the regulation of inhibitory neurotransmission (McLaughlin et al. 2014). Widespread overlapping of CB1Rs and GABAergic nerve endings suggests a key role for endocannabinoids in fine tuning of interneuron activity, which in turn regulates the net output of the ACC and OFC through direct and indirect synaptic interaction with cortical glutamatergic pyramidal cells. The ACC sends glutamatergic output projections to several cortical areas including motor and somatosensory cortices, as well as subcortical structures including the NAc and the VTA (Sesack et al. 1989; Uylings and van Eden 1990). It has been proposed that these latter 
projections may be crucial for mediating the regulatory function of the ACC during effort-based decision making (Walton et al. 2006). In addition to these areas, the OFC sends output projections to the serotonergic neurons in the dorsal raphe nucleus (Cavada et al. 2000; Roberts 2011; Vazquez-Borsetti et al. 2009) also, which suggests that the OFC may contribute to the regulation of serotonergic drive in the brain. Considering that interneurons exert a direct inhibitory influence on pyramidal neurons, the cannabinoid-induced suppression of presynaptic GABA release is expected to cause an increased pyramidal cell activity. This enhancement of ACC and OFC pyramidal cell activity should in turn lead to increased glutamate release in their target structures. Such an enhancement is in clear contradiction to the behavioral consequences of ACC and OFC lesions: Cannabinoid activation in the ACC and OFC and lesions of these areas (Rudebeck et al. 2006) induce similar deficits in effort-based and delay-based decision making, respectively. However, according to this mechanism, the target areas will receive a strengthened input from the ACC and OFC following local cannabinoid activation whereas the target areas are deprived from the ACC and OFC input following a lesion in the respective area. This apparent contradiction can be explained at least in three different ways. First, it has been shown that interneurons, particularly in the ACC, synchronize through gap junctions (Chang and Shyu 2014) and are responsible for the generation of oscillations in different frequencies (Whittington and Traub 2003). It is possible that despite the strengthened output activity, disruption of synchronized activity and oscillations necessary for optimal decision making might account for suboptimal decision making following cannabinoid system activation in the ACC and OFC. Second, cannabinoid system activation can lead to overactivation-induced disruption of frontal cortex function (Baiardi et al. 2007). Third, the interneurons might exert a more sophisticated control on the principal neurons output through a variety of synapses rather than a simply direct inhibition of principal neurons. Recently, there have been several studies demonstrating the importance of interneuroninterneuron interactions in the processing of cortical functions (Jiang et al. 2013; Pfeffer et al. 2013; Pi et al. 2013; Xu et al. 2013). These interactions, which consist of inhibition of inhibitory neurons, can lead to disinhibition of pyramidal cell activity by decreasing the net inhibitory drive on these neurons. Functional anatomical data indicates that interneurons frequently exhibit synaptic contacts with other interneurons in the mouse ACC (Wu et al. 2009), suggesting that neural circuits for disinhibition are present in this structure. If indeed the ACC and OFC cannabinoid effects on decision making

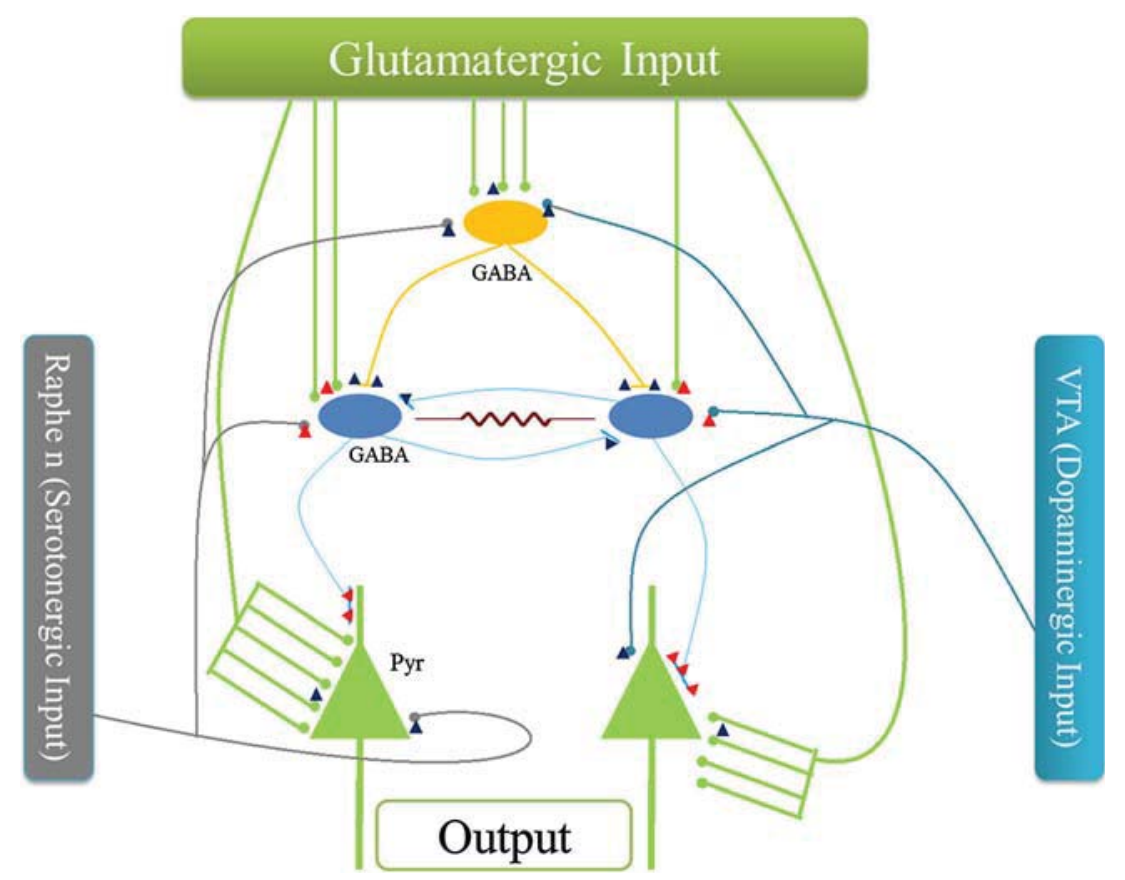

Fig 8 Schema of cannabinoid modulatory effects on frontal cortical circuits. A simplified schematic representation of neuronal circuitry in the ACC and OFC illustrating possible synapses that can be modulated by cannabinoids. Disinhibitory and inhibitory GABAergic interneurons are shown in orange and blue, respectively. Excitatory axonal ends are shown with small circles and inhibitory synaptic ends with a perpendicular line. Presynaptic CB1Rs are shown as small triangles. The color of the triangles represent the net effect of CB1R activation on the glutamatergic output with dark blue and red triangles signifying net inhibitory and facilitatory influence on the (subpopulation of) output pyramidal neurons. The electrical synapse between two interneurons is also depicted, since gap junctions between fast spiking interneurons play an important role in the generation of oscillations in various frequencies which might be modulated or disrupted by cannabinoid modulation. Note that synaptic ends of dopaminergic neurons are mostly excitatory as depicted. They can however also exert an inhibitory influence on some synapses, an effect that is dependent on the relative densities of D1 and D2-like receptors. Pyr; Pyramidal neuron 
involve disinhibitory mechanisms, it follows that the CB1R receptors would have to be localized predominately in GABAergic presynapses that contact other GABAergic, and not glutamatergic, cortical neurons. This is the case because depending on interneuron connectivity, CB1R-mediated suppression of GABA release may have the opposing effects: net enhancement of pyramidal neuron output when an interneuron to pyramidal neuron synapse is modulated or net inhibition of pyramidal neuron output when an interneuron to interneuron synapse is modulated. Figure 8 summarizes the possible mechanisms by which cannabinoids might modulate activity within the ACC and OFC. It has to be noted that the deficits induced by injection of cannabinoid agonist were in some cases even more severe than those induced by the lesion of the respective area. This might be explained by the fact that the lesion shuts down the functional connectivity of the area while in the current study the functional connectivity remains intact and can exert stronger influence on behavior using one or more of the possible mechanisms described above.

Finally, we observed sporadic colocalizations of CB1Rs on glutamatergic axonal ends in the ACC and OFC, suggesting that direct effects of cannabinoids on glutamatergic neurons may play a minor role in mediating behavioral deficits during effort-based and delay-based decision making. However, we cannot rule out the importance of the cannabinoid modulation of the ACC excitatory input during effort-based decision task. Indeed, CB1R-dependent impairments in locomotor, temperature homeostasis, and nociceptive functions have been shown to involve mostly glutamatergic and not GABAergic neural populations (Monory et al. 2007).

In summary, our experiments suggest that cannabinoid system is a major regulator of synaptic transmission in the ACC and OFC and plays a critical role in the balanced processing of effort and delay costs in cost-benefit decision making. A number of studies showed high CB1R binding and elevated levels of endogenous cannabinoids in schizophrenia (Zavitsanou et al. 2004) in the ACC and in alcohol dependence (Neumeister et al. 2012) in the ACC and OFC, disorders in which executive function and decision making have been compromised which implicates cannabinoid system abnormalities in the pathology of these disorders. Future work should determine further characteristics, including but not limited to dose-response profile, of endocannabinoid regulation of decision-making processes with possible clinical implications, and therapeutic potential of manipulating cannabinoid system function in a range of psychiatric disorders with impairments in decision making.

Acknowledgments This work was supported by The European Young Investigator Award (EURYI) grant PE0033-117106. Confocal microscopy analysis was performed at the "Servicio de Microscopia Analítica y de Alta Resolución en Biomedicina" (High Resolution Analytical Microscopy Services for Biomedicine) at the University of the Basque Country (UPV/EHU). The authors would like to thank Z. Fattahi and M. Moradi for their assistance in the experiments and C. Lamy and J.V. Lafuente for helpful comments on the earlier versions of this manuscript.

Conflicts of interest The authors declare no conflicts of interest.

\section{References}

Baiardi G, Ruiz AM, Beling A, Borgonovo J, Martinez G, Landa AI, Sosa MA, Gargiulo PA (2007) Glutamatergic ionotropic blockade within accumbens disrupts working memory and might alter the endocytic machinery in rat accumbens and prefrontal cortex. $\mathrm{J}$ Neural Transm 114:1519-1528

Bechara A, Damasio H, Damasio AR (2000) Emotion, decision making and the orbitofrontal cortex. Cereb Cortex 10:295-307

Bodor AL, Katona I, Nyiri G, Mackie K, Ledent C, Hajos N, Freund TF (2005) Endocannabinoid signaling in rat somatosensory cortex: laminar differences and involvement of specific interneuron types. J Neurosci Off J Soc Neurosci 25:6845-6856

Boomhower SR, Rasmussen EB, Doherty TS (2013) Impulsive-choice patterns for food in genetically lean and obese Zucker rats. Behav Brain Res 241:214-221

Castillo PE, Younts TJ, Chavez AE, Hashimotodani Y (2012) Endocannabinoid signaling and synaptic function. Neuron 76:70-81

Cavada C, Company T, Tejedor J, Cruz-Rizzolo RJ, Reinoso-Suarez F (2000) The anatomical connections of the macaque monkey orbitofrontal cortex. A review. Cereb Cortex 10:220-242

Chang WP, Shyu BC (2014) Anterior cingulate epilepsy: mechanisms and modulation. Front Integr Neurosci 7:104

Chiu CQ, Puente N, Grandes P, Castillo PE (2010) Dopaminergic modulation of endocannabinoid-mediated plasticity at GABAergic synapses in the prefrontal cortex. J Neurosci Off J Soc Neurosci 30: 7236-7248

Denk F, Walton ME, Jennings KA, Sharp T, Rushworth MF, Bannerman DM (2005) Differential involvement of serotonin and dopamine systems in cost-benefit decisions about delay or effort. Psychopharmacology 179:587-596

Domenici MR, Azad SC, Marsicano G, Schierloh A, Wotjak CT, Dodt HU, Zieglgansberger W, Lutz B, Rammes G (2006) Cannabinoid receptor type 1 located on presynaptic terminals of principal neurons in the forebrain controls glutamatergic synaptic transmission. J Neurosci Off J Soc Neurosci 26:5794-5799

Dougherty DM, Mathias CW, Dawes MA, Furr RM, Charles NE, Liguori A, Shannon EE, Acheson A (2013) Impulsivity, attention, memory, and decision-making among adolescent marijuana users. Psychopharmacology 226:307-319

Dubreucq S, Durand A, Matias I, Benard G, Richard E, Soria-Gomez E, Glangetas C, Groc L, Wadleigh A, Massa F, Bartsch D, Marsicano G, Georges F, Chaouloff F (2013) Ventral tegmental area cannabinoid type-1 receptors control voluntary exercise performance. Biol Psychiatry 73:895-903

Egerton A, Allison C, Brett RR, Pratt JA (2006) Cannabinoids and prefrontal cortical function: insights from preclinical studies. Neurosci Biobehav Rev 30:680-695

Egerton A, Brett RR, Pratt JA (2005) Acute delta9-tetrahydrocannabinolinduced deficits in reversal learning: neural correlates of affective inflexibility. Off Publ Am Coll Neuropsychopharmacology 30: 1895-1905

Egertova M, Elphick MR (2000) Localisation of cannabinoid receptors in the rat brain using antibodies to the intracellular C-terminal tail of CB. J Comp Neurol 422:159-171

Eggan SM, Lewis DA (2007) Immunocytochemical distribution of the cannabinoid CB1 receptor in the primate neocortex: a regional and laminar analysis. Cereb Cortex 17:175-191 
Ehlers CL, Slutske WS, Lind PA, Wilhelmsen KC (2007) Association between single nucleotide polymorphisms in the cannabinoid receptor gene (CNR1) and impulsivity in southwest California Indians. Twin research and human genetics : the official journal of the International Society for Twin Studies 10:805-811

Engeli S (2012) Central and peripheral cannabinoid receptors as therapeutic targets in the control of food intake and body weight. Handbook of experimental pharmacology: 357-81

Filbey FM, Schacht JP, Myers US, Chavez RS, Hutchison KE (2010) Individual and additive effects of the CNR1 and FAAH genes on brain response to marijuana cues. Neuropsychopharmacology 35 : 967-975

Fogaca MV, Aguiar DC, Moreira FA, Guimaraes FS (2012) The endocannabinoid and endovanilloid systems interact in the rat prelimbic medial prefrontal cortex to control anxiety-like behavior. Neuropharmacology 63:202-210

Ganon-Elazar E, Akirav I (2009) Cannabinoid receptor activation in the basolateral amygdala blocks the effects of stress on the conditioning and extinction of inhibitory avoidance. J Neurosci Off J Soc Neurosci 29:11078-11088

Grace AA (2000) Gating of information flow within the limbic system and the pathophysiology of schizophrenia. Brain Res Brain Res Rev 31:330-341

Grace AA, Floresco SB, Goto Y, Lodge DJ (2007) Regulation of firing of dopaminergic neurons and control of goal-directed behaviors. Trends Neurosci 30:220-227

Grant JE, Chamberlain SR, Schreiber L, Odlaug BL (2012) Neuropsychological deficits associated with cannabis use in young adults. Drug Alcohol Depend 121:159-162

Hadamitzky M, Feja M, Becker T, Koch M (2009) Effects of acute systemic administration of serotonin $2 \mathrm{~A} / \mathrm{C}$ receptor ligands in a delay-based decision-making task in rats. Behav Pharmacol 20: 415-423

Hampson RE, Sweatt AJ, Goonawardena AV, Song D, Chan RH, Marmarelis VZ, Berger TW, Deadwyler SA (2011) Memory encoding in hippocampal ensembles is negatively influenced by cannabinoid CB1 receptors. Behav Pharmacol 22:335-346

Hauber W, Sommer S (2009) Prefrontostriatal circuitry regulates effortrelated decision making. Cereb Cortex 19:2240-2247

Hermann D, Lemenager T, Gelbke J, Welzel H, Skopp G, Mann K (2009) Decision making of heavy cannabis users on the Iowa Gambling Task: stronger association with THC of hair analysis than with personality traits of the Tridimensional Personality Questionnaire. Eur Addict Res 15:94-98

Hernandez G, Oleson EB, Gentry RN, Abbas Z, Bernstein DL, Arvanitogiannis A, Cheer JF (2014) Endocannabinoids promote cocaine-induced impulsivity and its rapid dopaminergic correlates. Biol Psychiatry 75:487-498

Jacob W, Marsch R, Marsicano G, Lutz B, Wotjak CT (2012) Cannabinoid CB1 receptor deficiency increases contextual fear memory under highly aversive conditions and long-term potentiation in vivo. Neurobiol Learn Mem 98:47-55

Jiang X, Wang G, Lee AJ, Stornetta RL, Zhu JJ (2013) The organization of two new cortical interneuronal circuits. Nat Neurosci 16:210-218

Kano M, Ohno-Shosaku T, Hashimotodani Y, Uchigashima M, Watanabe M (2009) Endocannabinoid-mediated control of synaptic transmission. Physiol Rev 89:309-380

Katona I, Freund TF (2008) Endocannabinoid signaling as a synaptic circuit breaker in neurological disease. Nat Med 14:923-930

Kawamura Y, Fukaya M, Maejima T, Yoshida T, Miura E, Watanabe M, Ohno-Shosaku T, Kano M (2006) The CB1 cannabinoid receptor is the major cannabinoid receptor at excitatory presynaptic sites in the hippocampus and cerebellum. J Neurosci Off J Soc Neurosci 26: 2991-3001
Kennerley SW, Behrens TE, Wallis JD (2011) Double dissociation of value computations in orbitofrontal and anterior cingulate neurons. Nat Neurosci 14:1581-1589

Khani A (2014) Partially dissociable roles of OFC and ACC in stimulusguided and action-guided decision making. Journal of neurophysiology.

Lane SD, Cherek DR, Tcheremissine OV, Lieving LM, Pietras CJ (2005) Acute marijuana effects on human risk taking. Neuropsychopharmacology 30:800-809

Lee D, Rushworth MF, Walton ME, Watanabe M, Sakagami M (2007) Functional specialization of the primate frontal cortex during decision making. J Neurosci Off J Soc Neurosci 27:8170-8173

Lee MC, Ploner M, Wiech K, Bingel U, Wanigasekera V, Brooks J, Menon DK, Tracey I (2013) Amygdala activity contributes to the dissociative effect of cannabis on pain perception. Pain 154:124 134

Loflin M, Earleywine M, De Leo J, Hobkirk A (2014) Subtypes of attention deficit-hyperactivity disorder (ADHD) and cannabis use. Subst Use Misuse 49:427-434

Mai B, Sommer S, Hauber W (2012) Motivational states influence effortbased decision making in rats: the role of dopamine in the nucleus accumbens. Cogn Affect Behav Neurosci 12:74-84

Mailleux P, Vanderhaeghen JJ (1992) Distribution of neuronal cannabinoid receptor in the adult rat brain: a comparative receptor binding radioautography and in situ hybridization histochemistry. Neuroscience 48:655-668

Martin WJ, Coffin PO, Attias E, Balinsky M, Tsou K, Walker JM (1999) Anatomical basis for cannabinoid-induced antinociception as revealed by intracerebral microinjections. Brain Res 822:237-242

Matyas F, Yanovsky Y, Mackie K, Kelsch W, Misgeld U, Freund TF (2006) Subcellular localization of type 1 cannabinoid receptors in the rat basal ganglia. Neuroscience 137:337-361

McDonald J, Schleifer L, Richards JB, de Wit H (2003) Effects of THC on behavioral measures of impulsivity in humans. Neuropsychopharmacology 28:1356-1365

McLaughlin RJ, Hill MN, Gorzalka BB (2014) A critical role for prefrontocortical endocannabinoid signaling in the regulation of stress and emotional behavior. Neuroscience and biobehavioral reviews.

Miller AS, Sanudo-Pena MC, Walker JM (1998) Ipsilateral turning behavior induced by unilateral microinjections of a cannabinoid into the rat subthalamic nucleus. Brain Res 793:7-11

Mishima K, Fujii M, Aoo N, Yoshikawa T, Fukue Y, Honda Y, Egashira N, Iwasaki K, Shoyama Y, Fujiwara M (2002) The pharmacological characterization of attentional processes using a two-lever choice reaction time task in rats. Biol Pharm Bull 25:1570-1576

Monory K, Blaudzun H, Massa F, Kaiser N, Lemberger T, Schutz G, Wotjak CT, Lutz B, Marsicano G (2007) Genetic dissection of behavioural and autonomic effects of delta (9)-tetrahydrocannabinol in mice. PLoS Biol 5:e269

Moreira FA, Aguiar DC, Guimaraes FS (2007) Anxiolytic-like effect of cannabinoids injected into the rat dorsolateral periaqueductal gray. Neuropharmacology 52:958-965

Moreno M, Estevez AF, Zaldivar F, Montes JM, Gutierrez-Ferre VE, Esteban L, Sanchez-Santed F, Flores P (2012) Impulsivity differences in recreational cannabis users and binge drinkers in a university population. Drug Alcohol Depend 124:355-362

Navarrete M, Araque A (2008) Endocannabinoids mediate neuronastrocyte communication. Neuron 57:883-893

Neumeister A, Normandin MD, Murrough JW, Henry S, Bailey CR, Luckenbaugh DA, Tuit K, Zheng MQ, Galatzer-Levy IR, Sinha R, Carson RE, Potenza MN, Huang Y (2012) Positron emission tomography shows elevated cannabinoid CB1 receptor binding in men with alcohol dependence. Alcohol Clin Exp Res 36:2104-2109

Pattij T, Janssen MC, Schepers I, Gonzalez-Cuevas G, de Vries TJ, Schoffelmeer AN (2007) Effects of the cannabinoid CB1 receptor 
antagonist rimonabant on distinct measures of impulsive behavior in rats. Psychopharmacology 193:85-96

Paxinos G, Watson C (2007) The rat brain in stereotaxic coordinates, 6th edn. Academic Press/Elsevier, Amsterdam

Pfeffer CK, Xue M, He M, Huang ZJ, Scanziani M (2013) Inhibition of inhibition in visual cortex: the logic of connections between molecularly distinct interneurons. Nat Neurosci 16:1068-1076

Pi HJ, Hangya B, Kvitsiani D, Sanders JI, Huang ZJ, Kepecs A (2013) Cortical interneurons that specialize in disinhibitory control. Nature 503:521-524

Prevost C, Pessiglione M, Metereau E, Clery-Melin ML, Dreher JC (2010) Separate valuation subsystems for delay and effort decision costs. J Neurosci Off J Soc Neurosci 30:14080-14090

Reguero L, Puente N, Elezgarai I, Mendizabal-Zubiaga J, Canduela MJ, Buceta I, Ramos A, Suarez J, Rodriguez de Fonseca F, Marsicano G, Grandes P (2011) GABAergic and cortical and subcortical glutamatergic axon terminals contain $\mathrm{CB} 1$ cannabinoid receptors in the ventromedial nucleus of the hypothalamus. PLoS ONE 6:e26167

Robbe D, Montgomery SM, Thome A, Rueda-Orozco PE, McNaughton BL, Buzsaki G (2006) Cannabinoids reveal importance of spike timing coordination in hippocampal function. Nat Neurosci 9: 1526-1533

Roberts AC (2011) The importance of serotonin for orbitofrontal function. Biol Psychiatry 69:1185-1191

Rudebeck PH, Walton ME, Smyth AN, Bannerman DM, Rushworth MF (2006) Separate neural pathways process different decision costs. Nat Neurosci 9:1161-1168

Rushworth MF, Behrens TE, Rudebeck PH, Walton ME (2007) Contrasting roles for cingulate and orbitofrontal cortex in decisions and social behaviour. Trends Cogn Sci 11:168-176

Salamone JD, Cousins MS, Bucher S (1994) Anhedonia or anergia? Effects of haloperidol and nucleus accumbens dopamine depletion on instrumental response selection in a T-maze cost/benefit procedure. Behav Brain Res 65:221-229

Schindelin J, Arganda-Carreras I, Frise E, Kaynig V, Longair M, Pietzsch T, Preibisch S, Rueden C, Saalfeld S, Schmid B, Tinevez JY, White DJ, Hartenstein V, Eliceiri K, Tomancak P, Cardona A (2012) Fiji: an open-source platform for biological-image analysis. Nat Methods 9:676-682

Schulz S, Becker T, Nagel U, von Ameln-Mayerhofer A, Koch M (2013) Chronic co-administration of the cannabinoid receptor agonist WIN55,212-2 during puberty or adulthood reverses 3,4 methylenedioxymetamphetamine (MDMA)-induced deficits in recognition memory but not in effort-based decision making. Pharmacol Biochem Behav 106:91-100

Schweimer J, Hauber W (2005) Involvement of the rat anterior cingulate cortex in control of instrumental responses guided by reward expectancy. Learn Mem 12:334-342

Schweimer J, Hauber W (2006) Dopamine D1 receptors in the anterior cingulate cortex regulate effort-based decision making. Learn Mem 13:777-782

Sesack SR, Deutch AY, Roth RH, Bunney BS (1989) Topographical organization of the efferent projections of the medial prefrontal cortex in the rat: an anterograde tract-tracing study with Phaseolus vulgaris leucoagglutinin. J Comp Neurol 290:213-242

Sink KS, Vemuri VK, Olszewska T, Makriyannis A, Salamone JD (2008) Cannabinoid CB1 antagonists and dopamine antagonists produce different effects on a task involving response allocation and effort- related choice in food-seeking behavior. Psychopharmacology 196: 565-574

Solowij N, Jones KA, Rozman ME, Davis SM, Ciarrochi J, Heaven PC, Pesa N, Lubman DI, Yucel M (2012) Reflection impulsivity in adolescent cannabis users: a comparison with alcohol-using and non-substance-using adolescents. Psychopharmacology 219:575586

Svizenska I, Dubovy P, Sulcova A (2008) Cannabinoid receptors 1 and 2 (CB1 and CB2), their distribution, ligands and functional involvement in nervous system structures - a short review. Pharmacol Biochem Behav 90:501-511

Tsou K, Brown S, Sanudo-Pena MC, Mackie K, Walker JM (1998) Immunohistochemical distribution of cannabinoid CB1 receptors in the rat central nervous system. Neuroscience 83:393-411

Uylings HB, van Eden CG (1990) Qualitative and quantitative comparison of the prefrontal cortex in rat and in primates, including humans. Prog Brain Res 85:31-62

Vazquez-Borsetti P, Cortes R, Artigas F (2009) Pyramidal neurons in rat prefrontal cortex projecting to ventral tegmental area and dorsal raphe nucleus express 5-HT2A receptors. Cereb Cortex 19:16781686

Walton ME, Croxson PL, Rushworth MF, Bannerman DM (2005) The mesocortical dopamine projection to anterior cingulate cortex plays no role in guiding effort-related decisions. Behav Neurosci 119: 323-328

Walton ME, Kennerley SW, Bannerman DM, Phillips PE, Rushworth MF (2006) Weighing up the benefits of work: behavioral and neural analyses of effort-related decision making. Neural Netw 19:13021314

Webb RH, Dorey CK (1995) The pixelated image. In: Pawley JB (ed) Handbook of Biological Confocal Microscopy. Plenum Press, New York, pp 55-67

Whitlow CT, Liguori A, Livengood LB, Hart SL, Mussat-Whitlow BJ, Lamborn CM, Laurienti PJ, Porrino LJ (2004) Long-term heavy marijuana users make costly decisions on a gambling task. Drug Alcohol Depend 76:107-111

Whittington MA, Traub RD (2003) Interneuron diversity series: inhibitory interneurons and network oscillations in vitro. Trends Neurosci 26:676-682

Wiskerke J, Stoop N, Schetters D, Schoffelmeer AN, Pattij T (2011) Cannabinoid $\mathrm{CB} 1$ receptor activation mediates the opposing effects of amphetamine on impulsive action and impulsive choice. PLoS ONE 6:e25856

Wu LJ, Li X, Chen T, Ren M, Zhuo M (2009) Characterization of intracortical synaptic connections in the mouse anterior cingulate cortex using dual patch clamp recording. Mol Brain Res 2:32

Xu H, Jeong HY, Tremblay R, Rudy B (2013) Neocortical somatostatinexpressing GABAergic interneurons disinhibit the thalamorecipient layer 4. Neuron 77:155-167

Zavitsanou K, Garrick T, Huang XF (2004) Selective antagonist [3H] SR141716A binding to cannabinoid CB1 receptors is increased in the anterior cingulate cortex in schizophrenia. Prog NeuroPsychopharmacol Biol Psychiatry 28:355-360

Zeeb FD, Floresco SB, Winstanley CA (2010) Contributions of the orbitofrontal cortex to impulsive choice: interactions with basal levels of impulsivity, dopamine signalling, and reward-related cues. Psychopharmacology 211:87-98 